\title{
Ordering Chemical Graphs by Sombor Indices and Its Applications
}

\author{
Hechao Liu ${ }^{1}$, Lihua You ${ }^{1, *}$, Yufei Huang ${ }^{2}$ \\ ${ }^{1}$ School of Mathematical Sciences, South China Normal University, \\ Guangzhou, 510631, P. R. China \\ hechaoliu@m.scnu.edu.cn, ylhua@scnu.edu.cn \\ ${ }^{2}$ Department of Mathematics Teaching, Guangzhou Civil Aviation College, \\ Guangzhou, 510403, P. R. China \\ fayger@qq. com
}

(Received March 17, 2021)

\begin{abstract}
Topological indices are a class of numerical invariants that predict certain physical and chemical properties of molecules. Recently, two novel topological indices, named as Sombor index and reduced Sombor index, were introduced by Gutman, defined as

$$
\begin{gathered}
S O(G)=\sum_{u v \in E(G)} \sqrt{d_{G}^{2}(u)+d_{G}^{2}(v)}, \\
S O_{r e d}(G)=\sum_{u v \in E(G)} \sqrt{\left(d_{G}(u)-1\right)^{2}+\left(d_{G}(v)-1\right)^{2}},
\end{gathered}
$$

where $d_{G}(u)$ denotes the degree of vertex $u$ in $G$.

In this paper, our aim is to order the chemical trees, chemical unicyclic graphs, chemical bicyclic graphs and chemical tricyclic graphs with respect to Sombor index and reduced Sombor index. We determine the first fourteen minimum chemical trees, the first four minimum chemical unicyclic graphs, the first three minimum chemical bicyclic graphs, the first seven minimum chemical tricyclic graphs. At last, we consider the applications of reduced Sombor index to octane isomers.
\end{abstract}

\footnotetext{
*Corresponding author
} 


\section{Introduction}

Let $G$ be a simple connected graph with vertex set $V(G)$ and edge set $E(G)$. Denote by $N_{G}(u)$ the set of vertices adjacent to $u$ in $G$ for every $u \in V(G)$. The degree $d_{G}(u)$ of $u$ in $G$ is the cardinality of $N_{G}(u)$. Let $\Delta(G)$ (or simply $\Delta$ ) be the maximum degree of $G$. Let $n_{i}(G)$ (or simply $n_{i}$ ) be the number of vertices with degree $i$ in $G$. Denote by $m_{i, j}(G)$ the numbers of edges connected a vertex with degree $i$ and a vertex with degree $j$ in $G$. In this paper, all notations and terminologies used but not defined can refer to the textbook [4].

The Sombor index $\left(S O(G)\right.$ for short) and reduced Sombor index $\left(S O_{\text {red }}(G)\right.$ for short $)$ of a graph $G$ are defined as [10]

$$
\begin{gathered}
S O(G)=\sum_{u v \in E(G)} \sqrt{d_{G}^{2}(u)+d_{G}^{2}(v)}, \\
S O_{r e d}(G)=\sum_{u v \in E(G)} \sqrt{\left(d_{G}(u)-1\right)^{2}+\left(d_{G}(v)-1\right)^{2}} .
\end{gathered}
$$

Shortly after, Deng et al. [9] determined the maximum Sombor indices of chemical trees. Cruz et al. [5] determined the extremal values of some chemical graphs. Also, Redžepović [24] studied chemical applicability of Sombor indices. Milovanović et al. [23] considered the bounds of Sombor indices and the relations between Sombor indices and other indices. Furthermore, Liu et al. [22] obtained some bounds for reduced Sombor index of graphs with given several parameters and some special graphs, they also obtained the the expected values of reduced Sombor index in random polyphenyl chains, the bounds of reduced Sombor spectrum radius and energy. For more details of Sombor indices, we refer to $[2,8,10,11,18,25,26]$.

The chemical graph is a graph with $d_{G}(u) \leq 4$ for all $u \in V(G)$. Ordering chemical graphs by some topological indices is an interesting problem. Ghalavand and Ashrafi had done a lot of work on ordering chemical graphs by some topological indices such as Wiener polarity index [3], sum exdeg index [12], total irregularity [13], forgotten coindex [14], Randić index and sum-connectivity index [15] and hyper-Zagreb index [16]. For more related papers can be find in $[1,17,19]$ and references cited therein.

Motivated by $[15,17]$, our aim is to consider the similar issues regarding (reduced) Sombor index. In this paper, we determine the first fourteen minimum chemical trees, the first four minimum chemical unicyclic graphs, the first three minimum chemical bi- 
cyclic graphs, the first seven minimum chemical tricyclic graphs. At last, we consider the applications of reduced Sombor index to octane isomers.

\section{Preliminaries}

Here are some important transformations that will be used in the proof of main results.

Lemma 2.1 Let $G_{0}$ be a connected graph with vertices $u_{1}, u_{2}, u_{3}, u_{4}\left(d_{G_{0}}\left(u_{1}\right)=1, d_{G_{0}}\left(u_{2}\right)\right.$ $=2, d_{G_{0}}\left(u_{3}\right)=3$ or $\left.4, d_{G_{0}}\left(u_{4}\right)=1,\left\{u_{1} u_{2}, u_{3} u_{4}\right\} \subseteq E\left(G_{0}\right)\right)$. Suppose that $P=v_{1} v_{2} \cdots v_{l}$ is a path. Denote by $G_{1}$ the graph gotten from $G_{0}, P$ by attaching vertices $u_{1} v_{1}$. Let $G_{2}=G_{1}-u_{1} v_{1}+u_{4} v_{1}$. Then $S O\left(G_{1}\right)>S O\left(G_{2}\right)$ and $S O_{\text {red }}\left(G_{1}\right)>S O_{\text {red }}\left(G_{2}\right)$.

Proof. By the definition of Sombor index, we have

$$
\begin{aligned}
S O\left(G_{2}\right)-S O\left(G_{1}\right) & =\sqrt{1^{2}+2^{2}}+\sqrt{d_{G_{0}}^{2}\left(u_{3}\right)+2^{2}}-\left[\sqrt{2^{2}+2^{2}}+\sqrt{d_{G_{0}}^{2}\left(u_{3}\right)+1^{2}}\right] \\
& =\sqrt{5}+\sqrt{d_{G_{0}}^{2}\left(u_{3}\right)+4}-2 \sqrt{2}-\sqrt{d_{G_{0}}^{2}\left(u_{3}\right)+1^{2}}
\end{aligned}
$$

If $d_{G_{0}}^{2}\left(u_{3}\right)=3$, then $S O\left(G_{2}\right)-S O\left(G_{1}\right)=\sqrt{5}+\sqrt{13}-2 \sqrt{2}-\sqrt{10}<0$.

If $d_{G_{0}}^{2}\left(u_{3}\right)=4$, then $S O\left(G_{2}\right)-S O\left(G_{1}\right)=\sqrt{5}+\sqrt{20}-2 \sqrt{2}-\sqrt{17}<0$.

Therefore $S O\left(G_{1}\right)>S O\left(G_{2}\right)$. In a similar way, we also have $S O_{\text {red }}\left(G_{1}\right)>S O_{\text {red }}\left(G_{2}\right)$. This completes the proof.

Lemma 2.2 Let $G_{0}, G$ be two connected graphs with vertices $x \in V\left(G_{0}\right), y \in V(G)$ ( $d_{G_{0}}(x)=1$ or 2 and $d_{G_{0}}(y)=2$ or 3$)$. Suppose that $P_{1}=u_{1} u_{2} \cdots u_{k}$ and $P_{2}=v_{1} v_{2} \cdots v_{l}$ are two paths. Denote by $G_{1}$ the graph gotten from $G_{0}, G, P_{1}$ and $P_{2}$ by attaching vertices $v_{1} x, u_{1} x$ and $u_{k} y$. Let $G_{2}=G_{1}-\left\{u_{1} x, u_{k} y\right\}+\left\{x y, u_{1} v_{l}\right\}$. Then $S O\left(G_{1}\right)>S O\left(G_{2}\right)$ and $S O_{\text {red }}\left(G_{1}\right)>S O_{\text {red }}\left(G_{2}\right)$.

Proof. We consider the following two cases.

Case 1. $l=1$.

$$
\begin{aligned}
S O\left(G_{2}\right)-S O\left(G_{1}\right)= & \sqrt{1^{2}+2^{2}}+\sqrt{\left(d_{G_{0}}(x)+2\right)^{2}+\left(d_{G_{0}}(y)+1\right)^{2}} \\
& -\left[\sqrt{\left(d_{G_{0}}(x)+2\right)^{2}+1^{2}}+\sqrt{\left(d_{G_{0}}(y)+1\right)^{2}+2^{2}}\right] .
\end{aligned}
$$

Since $d_{G_{0}}(x)=1$ or 2 and $d_{G_{0}}(y)=2$ or 3 .

If $d_{G_{0}}(x)=1$ and $d_{G_{0}}(y)=2$, then $S O\left(G_{2}\right)-S O\left(G_{1}\right)=\sqrt{5}+3 \sqrt{2}-\sqrt{10}-\sqrt{13}<0$.

If $d_{G_{0}}(x)=1$ and $d_{G_{0}}(y)=3$, then $S O\left(G_{2}\right)-S O\left(G_{1}\right)=\sqrt{5}+5-\sqrt{10}-\sqrt{20}<0$. 
If $d_{G_{0}}(x)=2$ and $d_{G_{0}}(y)=2$, then $S O\left(G_{2}\right)-S O\left(G_{1}\right)=\sqrt{5}+5-\sqrt{17}-\sqrt{13}<0$.

If $d_{G_{0}}(x)=2$ and $d_{G_{0}}(y)=3$, then $S O\left(G_{2}\right)-S O\left(G_{1}\right)=\sqrt{5}+4 \sqrt{2}-\sqrt{17}-\sqrt{20}<0$.

Case 2. $l \geq 2$.

$$
\begin{aligned}
S O\left(G_{2}\right)-S O\left(G_{1}\right)= & \sqrt{2^{2}+2^{2}}+\sqrt{\left(d_{G_{0}}(x)+2\right)^{2}+\left(d_{G_{0}}(y)+1\right)^{2}} \\
& -\left[\sqrt{\left(d_{G_{0}}(x)+2\right)^{2}+2^{2}}+\sqrt{\left(d_{G_{0}}(y)+1\right)^{2}+2^{2}}\right] .
\end{aligned}
$$

Since $d_{G_{0}}(x)=1$ or 2 and $d_{G_{0}}(y)=2$ or 3 .

If $d_{G_{0}}(x)=1$ and $d_{G_{0}}(y)=2$, then $S O\left(G_{2}\right)-S O\left(G_{1}\right)=2 \sqrt{2}+3 \sqrt{2}-\sqrt{13}-\sqrt{13}<0$.

If $d_{G_{0}}(x)=1$ and $d_{G_{0}}(y)=3$, then $S O\left(G_{2}\right)-S O\left(G_{1}\right)=2 \sqrt{2}+5-\sqrt{13}-\sqrt{20}<0$.

If $d_{G_{0}}(x)=2$ and $d_{G_{0}}(y)=2$, then $S O\left(G_{2}\right)-S O\left(G_{1}\right)=2 \sqrt{2}+5-\sqrt{20}-\sqrt{13}<0$.

If $d_{G_{0}}(x)=2$ and $d_{G_{0}}(y)=3$, then $S O\left(G_{2}\right)-S O\left(G_{1}\right)=2 \sqrt{2}+4 \sqrt{2}-\sqrt{20}-\sqrt{20}<0$.

Therefore $S O\left(G_{1}\right)>S O\left(G_{2}\right)$. In a similar way, we also have $S O_{\text {red }}\left(G_{1}\right)>S O_{\text {red }}\left(G_{2}\right)$. This completes the proof.

Lemma 2.3 Let $G_{0}$ be a connected graph with the vertex $x\left(d_{G_{0}}(x)=1\right.$ or 2$)$. Suppose that $P_{1}=u_{1} u_{2} \cdots u_{k}$ and $P_{2}=v_{1} v_{2} \cdots v_{l}$ are two paths. Denote by $G_{1}$ the graph gotten from $G_{0}, P_{1}$ and $P_{2}$ by attaching vertices $u_{1} x$ and $v_{1} x$. Let $G_{2}=G_{1}-u_{1} x+u_{1} v_{l}$. Then $S O\left(G_{1}\right)>S O\left(G_{2}\right)$ and $S O_{\text {red }}\left(G_{1}\right)>S O_{\text {red }}\left(G_{2}\right)$.

Proof. Let $d_{G_{0}}(x)=t=1$ or $2, N_{G_{0}}(x)=\left\{z_{1}, z_{2}, \cdots, z_{t}\right\}, d_{G_{0}}\left(z_{i}\right)=d_{i}, 1 \leq i \leq t$.

Case 1. $k=l=1$.

$$
\begin{aligned}
S O\left(G_{2}\right)-S O\left(G_{1}\right)= & \sqrt{1^{2}+2^{2}}+\sqrt{(t+1)^{2}+2^{2}}+\sum_{i=1}^{t} \sqrt{d_{i}^{2}+(t+1)^{2}} \\
& -\left[\sqrt{(t+2)^{2}+1^{2}}+\sqrt{(t+2)^{2}+1^{2}}+\sum_{i=1}^{t} \sqrt{d_{i}^{2}+(t+2)^{2}}\right] \\
< & \sqrt{5}+\sqrt{(t+1)^{2}+2^{2}}-2 \sqrt{(t+2)^{2}+1^{2}}
\end{aligned}
$$

If $t=1$, then $S O\left(G_{2}\right)-S O\left(G_{1}\right)<\sqrt{5}+2 \sqrt{2}-2 \sqrt{10}<0$.

If $t=2$, then $S O\left(G_{2}\right)-S O\left(G_{1}\right)<\sqrt{5}+\sqrt{13}-2 \sqrt{17}<0$.

Case 2. $k=1, l \geq 2$.

$$
\begin{aligned}
S O\left(G_{2}\right)-S O\left(G_{1}\right) & <2 \sqrt{2}+\sum_{i=1}^{t} \sqrt{d_{i}^{2}+(t+1)^{2}}-\left[\sqrt{(t+2)^{2}+1^{2}}+\sum_{i=1}^{t} \sqrt{d_{i}^{2}+(t+2)^{2}}\right] \\
& <2 \sqrt{2}-\sqrt{(t+2)^{2}+1^{2}} .
\end{aligned}
$$

If $t=1$, then $S O\left(G_{2}\right)-S O\left(G_{1}\right)<2 \sqrt{2}-\sqrt{10}<0$. 
If $t=2$, then $S O\left(G_{2}\right)-S O\left(G_{1}\right)<2 \sqrt{2}-\sqrt{17}<0$.

Case 3. $k \geq 2, l=1$.

The conclusion holds from Case 2 and symmetry.

Case $4 . k \geq 2, l \geq 2$.

$$
\begin{aligned}
S O\left(G_{2}\right)-S O\left(G_{1}\right)= & 2 \sqrt{2^{2}+2^{2}}+\sqrt{(t+1)^{2}+2^{2}}+\sum_{i=1}^{t} \sqrt{d_{i}^{2}+(t+1)^{2}} \\
& -\left[\sqrt{1^{2}+2^{2}}+2 \sqrt{(t+2)^{2}+2^{2}}+\sum_{i=1}^{t} \sqrt{d_{i}^{2}+(t+2)^{2}}\right] \\
< & 4 \sqrt{2}+\sqrt{(t+1)^{2}+2^{2}}-\sqrt{5}-2 \sqrt{(t+2)^{2}+2^{2}} .
\end{aligned}
$$

If $t=1$, then $S O\left(G_{2}\right)-S O\left(G_{1}\right)<6 \sqrt{2}-2 \sqrt{13}-\sqrt{5}<0$.

If $t=2$, then $S O\left(G_{2}\right)-S O\left(G_{1}\right)<\sqrt{13}+4 \sqrt{2}-5 \sqrt{5}<0$.

Therefore $S O\left(G_{1}\right)>S O\left(G_{2}\right)$. In a similar way, we also have $S O_{\text {red }}\left(G_{1}\right)>S O_{\text {red }}\left(G_{2}\right)$.

This completes the proof.

Lemma 2.4 Let $G_{0}$ be a connected graph with vertices $x$ and $y\left(d_{G_{0}}(x)=2\right.$ or 3 , $d_{G_{0}}(y)=2$ or 3$)$. Suppose that $P_{1}=u_{1} u_{2} \cdots u_{k}$ and $P_{2}=v_{1} v_{2} \cdots v_{l}$ are two paths. Denote by $G_{1}$ the graph gotten from $G_{0}, P_{1}$ and $P_{2}$ by attaching vertices $u_{1} x$ and $v_{1} y$. Let $G_{2}=G_{1}-u_{1} x+u_{1} v_{l}$. Then $S O\left(G_{1}\right)>S O\left(G_{2}\right)$ and $S O_{\text {red }}\left(G_{1}\right)>S O_{\text {red }}\left(G_{2}\right)$.

Proof. Let $d_{G_{0}}(x)=t=2$ or $3, N_{G_{0}}(x)=\left\{z_{1}, z_{2}, \cdots, z_{t}\right\}, d_{G_{0}}\left(z_{i}\right)=d_{i}, 1 \leq i \leq t$.

Case 1. $k=l=1$.

$$
\begin{aligned}
& S O\left(G_{2}\right)-S O\left(G_{1}\right)=\sqrt{1^{2}+2^{2}}+\sqrt{\left(d_{G_{0}}(y)+1\right)^{2}+2^{2}}+\sum_{i=1}^{t} \sqrt{d_{i}^{2}+t^{2}} \\
& -\left[\sqrt{\left(d_{G_{0}}(y)+1\right)^{2}+1^{2}}+\sqrt{(t+1)^{2}+1^{2}}+\sum_{i=1}^{t} \sqrt{d_{i}^{2}+(t+1)^{2}}\right] \\
& <\sqrt{5}+\sqrt{\left(d_{G_{0}}(y)+1\right)^{2}+4}-\left[\sqrt{\left(d_{G_{0}}(y)+1\right)^{2}+1^{2}}+\sqrt{(t+1)^{2}+1^{2}}\right] .
\end{aligned}
$$

Since $d_{G_{0}}(y)=2$ or 3 and $t=2$ or 3 .

If $d_{G_{0}}(y)=2$ and $t=2$, then $S O\left(G_{2}\right)-S O\left(G_{1}\right)<\sqrt{5}+\sqrt{13}-\sqrt{10}-\sqrt{10}<0$.

If $d_{G_{0}}(y)=2$ and $t=3$, then $S O\left(G_{2}\right)-S O\left(G_{1}\right)<\sqrt{5}+\sqrt{13}-\sqrt{10}-\sqrt{17}<0$.

If $d_{G_{0}}(y)=3$ and $t=2$, then $S O\left(G_{2}\right)-S O\left(G_{1}\right)<\sqrt{5}+\sqrt{20}-\sqrt{10}-\sqrt{17}<0$.

If $d_{G_{0}}(y)=3$ and $t=3$, then $S O\left(G_{2}\right)-S O\left(G_{1}\right)<\sqrt{5}+\sqrt{20}-\sqrt{17}-\sqrt{17}<0$. 
Case 2. $k=1, l \geq 2$.

$$
\begin{aligned}
S O\left(G_{2}\right)-S O\left(G_{1}\right) & =\sqrt{2^{2}+2^{2}}+\sum_{i=1}^{t} \sqrt{d_{i}^{2}+t^{2}}-\left[\sqrt{(t+1)^{2}+1^{2}}+\sum_{i=1}^{t} \sqrt{d_{i}^{2}+(t+1)^{2}}\right] \\
& <2 \sqrt{2}-\sqrt{(t+1)^{2}+1^{2}}
\end{aligned}
$$

Since $t=2$ or 3 .

If $t=2$, then $S O\left(G_{2}\right)-S O\left(G_{1}\right)<2 \sqrt{2}-\sqrt{10}<0$.

If $t=3$, then $S O\left(G_{2}\right)-S O\left(G_{1}\right)<2 \sqrt{2}-\sqrt{17}<0$.

Case 3. $k \geq 2, l=1$.

$$
\begin{aligned}
& S O\left(G_{2}\right)-S O\left(G_{1}\right)=\sqrt{2^{2}+2^{2}}+\sqrt{\left(d_{G_{0}}(y)+1\right)^{2}+2^{2}}+\sum_{i=1}^{t} \sqrt{d_{i}^{2}+t^{2}} \\
& -\left[\sqrt{\left(d_{G_{0}}(y)+1\right)^{2}+1^{2}}+\sqrt{(t+1)^{2}+2^{2}}+\sum_{i=1}^{t} \sqrt{d_{i}^{2}+(t+1)^{2}}\right] \\
& <2 \sqrt{2}+\sqrt{\left(d_{G_{0}}(y)+1\right)^{2}+4}-\left[\sqrt{\left(d_{G_{0}}(y)+1\right)^{2}+1^{2}}+\sqrt{(t+1)^{2}+4}\right] .
\end{aligned}
$$

Since $d_{G_{0}}(y)=2$ or 3 and $t=2$ or 3 .

If $d_{G_{0}}(y)=2$ and $t=2$, then $S O\left(G_{2}\right)-S O\left(G_{1}\right)<2 \sqrt{2}+\sqrt{13}-\sqrt{10}-\sqrt{13}<0$.

If $d_{G_{0}}(y)=2$ and $t=3$, then $S O\left(G_{2}\right)-S O\left(G_{1}\right)<2 \sqrt{2}+\sqrt{13}-\sqrt{10}-\sqrt{20}<0$.

If $d_{G_{0}}(y)=3$ and $t=2$, then $S O\left(G_{2}\right)-S O\left(G_{1}\right)<2 \sqrt{2}+\sqrt{20}-\sqrt{17}-\sqrt{13}<0$.

If $d_{G_{0}}(y)=3$ and $t=3$, then $S O\left(G_{2}\right)-S O\left(G_{1}\right)<2 \sqrt{2}+\sqrt{20}-\sqrt{17}-\sqrt{20}<0$.

Case $4 . k \geq 2, l \geq 2$.

$$
\begin{aligned}
S O\left(G_{2}\right)-S O\left(G_{1}\right)= & 2 \sqrt{2^{2}+2^{2}}+\sum_{i=1}^{t} \sqrt{d_{i}^{2}+t^{2}} \\
& -\left[\sqrt{1^{2}+2^{2}}+\sqrt{(t+1)^{2}+2^{2}}+\sum_{i=1}^{t} \sqrt{d_{i}^{2}+(t+1)^{2}}\right] \\
< & 4 \sqrt{2}-\sqrt{5}-\sqrt{(t+1)^{2}+4}
\end{aligned}
$$

Since $t=2$ or 3 .

If $t=2$, then $S O\left(G_{2}\right)-S O\left(G_{1}\right)<4 \sqrt{2}-\sqrt{5}-\sqrt{13}<0$.

If $t=3$, then $S O\left(G_{2}\right)-S O\left(G_{1}\right)<4 \sqrt{2}-\sqrt{5}-\sqrt{20}<0$.

Therefore $S O\left(G_{1}\right)>S O\left(G_{2}\right)$. In a similar way, we also have $S O_{\text {red }}\left(G_{1}\right)>S O_{\text {red }}\left(G_{2}\right)$. This completes the proof. 


\section{$3 \quad$ Main results}

Denote by $C T_{n}, C U_{n}, C B_{n}, C T G_{n}$ the set of chemical trees, chemical unicyclic graphs, chemical bicyclic graphs, chemical tricyclic graphs with $n$ vertices, respectively. If $G$ has $t_{i}$ vertices of degree $d_{i}$ for $1 \leq i \leq s$, then we denote the degree sequence of $G$ as $D(G) \triangleq\left(d_{1}^{t_{1}}, d_{2}^{t_{2}}, \cdots, d_{s}^{t_{s}}\right)$, where $\sum_{i=1}^{s} t_{i}=n$.

\subsection{Chemical trees}

Let $\Phi(n)=\left\{T \in\left(4^{1}, 2^{n-5}, 1^{4}\right) \mid m_{1,2}(T)=m_{2,4}(T)=4, m_{1,4}(T)=0, m_{2,2}(T)=n-9\right\}$, $n \geq 9$, and $\Omega(n)=\left\{T \in\left(3^{3}, 2^{n-8}, 1^{5}\right) \mid m_{1,2}(T)=m_{2,3}(T)=5, m_{1,3}(T)=0, m_{3,3}(T)=\right.$ $\left.2, m_{2,2}(T)=n-13\right\}, n \geq 13$.

If $T \in \Phi(n)$, then

$$
\begin{gathered}
S O(T)=2 \sqrt{2} n+12 \sqrt{5}-18 \sqrt{2} \approx 2 \sqrt{2} n+1.376971607 \\
S O_{\text {red }}(T)=\sqrt{2} n+4+4 \sqrt{10}-9 \sqrt{2} \approx \sqrt{2} n+3.921188579 .
\end{gathered}
$$

If $T \in \Omega(n)$, then

$$
\begin{gathered}
S O(T)=2 \sqrt{2} n+5 \sqrt{5}+5 \sqrt{13}-20 \sqrt{2} \approx 2 \sqrt{2} n+0.923825017 \\
S O_{\text {red }}(T)=\sqrt{2} n+5+5 \sqrt{5}-9 \sqrt{2} \approx \sqrt{2} n+3.452417826
\end{gathered}
$$

Recall that $n_{i}$ is the numbers of vertices with degree $i$ in $G$. If $G \in C T_{n}$, since $\sum_{i=1}^{4} n_{i}=n$ and $n_{1}+2 n_{2}+3 n_{3}+4 n_{4}=2(n-1)$, then $n_{1}=n_{3}+2 n_{4}+2$ and $n_{2}=n-2 n_{3}-3 n_{4}-2$.

Theorem 3.1 Let $T^{*} \in C T_{n}(n \geq 9), \Delta\left(T^{*}\right)=4$. If $T^{*} \notin \Phi(n)$, then there exists $T \in \Phi(n)$, such that $S O(T)<S O\left(T^{*}\right)$ and $S O_{\text {red }}(T)<S O_{\text {red }}\left(T^{*}\right)$.

Proof. We consider the following two cases.

Case 1. If $T^{*} \in\left(4^{1}, 2^{n-5}, 1^{4}\right)$, then $T^{*}$ meets at least one of the following conditions $m_{1,2}\left(T^{*}\right) \neq 4, m_{2,4}\left(T^{*}\right) \neq 4, m_{1,4}\left(T^{*}\right) \neq 0, m_{2,2}\left(T^{*}\right) \neq n-9$, i.e., $m_{1,2}\left(T^{*}\right)<4, m_{2,4}\left(T^{*}\right)<$ $4, m_{1,4}\left(T^{*}\right)>0, m_{2,2}\left(T^{*}\right)>n-9$. By the transformation of Lemma 2.1, we can obtain a chemical tree $T \in \Phi(n)$, so we have $S O(T)<S O\left(T^{*}\right)$.

Case 2. If $T^{*} \notin\left(4^{1}, 2^{n-5}, 1^{4}\right)$. By the transformation of Lemma 2.3, we can obtain a chemical tree $T \in\left(4^{1}, 2^{n-5}, 1^{4}\right)$. If $T \in \Phi(n)$, by Lemma 2.3 , we have $S O(T)<S O\left(T^{*}\right)$. If $T \notin \Phi(n)$, we are back to Case 1 and the conclusion holds.

Therefore $S O(T)<S O\left(T^{*}\right)$. In a similar way, we also have $S O_{\text {red }}(T)<S O_{\text {red }}\left(T^{*}\right)$. This completes the proof. 
Theorem 3.2 Let $T^{*} \in C T_{n}(n \geq 13), \Delta\left(T^{*}\right)=3, n_{3}\left(T^{*}\right) \geq 3$. If $T^{*} \notin \Omega(n)$, then there exists $T \in \Omega(n)$, such that $S O(T)<S O\left(T^{*}\right)$ and $S O_{\text {red }}(T)<S O_{\text {red }}\left(T^{*}\right)$.

Proof. We consider the following two cases.

Case 1. If $T^{*} \in\left(3^{3}, 2^{n-8}, 1^{5}\right)$, then $T^{*}$ meets at least one of the following conditions $m_{1,2}\left(T^{*}\right) \neq 5, m_{2,3}\left(T^{*}\right) \neq 5, m_{1,3}\left(T^{*}\right) \neq 0, m_{3,3}\left(T^{*}\right) \neq 2, m_{2,2}\left(T^{*}\right) \neq n-13$, i.e., $m_{1,2}\left(T^{*}\right)<5, m_{2,3}\left(T^{*}\right)>5, m_{1,3}\left(T^{*}\right)>0, m_{3,3}\left(T^{*}\right)<2, m_{2,2}\left(T^{*}\right)>n-13$. By the transformation of Lemma 2.1 and Lemma 2.2, we can obtain a chemical tree $T \in \Omega(n)$, so we have $S O(T)<S O\left(T^{*}\right)$.

Case 2. If $T^{*} \notin\left(3^{3}, 2^{n-8}, 1^{5}\right)$, since $n_{1}\left(T^{*}\right)=n_{3}\left(T^{*}\right)+2$ and $n_{3}\left(T^{*}\right) \geq 3$, then $n_{3}\left(T^{*}\right) \geq 4$. By the transformation of Lemma 2.3 , we can obtain a chemical tree $T \in\left(3^{3}, 2^{n-8}, 1^{5}\right)$. If $T \in \Omega(n)$, by Lemma 2.3 , we have $S O(T)<S O\left(T^{*}\right)$. If $T \notin \Omega(n)$, we are back to Case 1 and the conclusion holds.

Therefore $S O(T)<S O\left(T^{*}\right)$. In a similar way, we also have $S O_{\text {red }}(T)<S O_{\text {red }}\left(T^{*}\right)$. This completes the proof.

In what follows, we determine the extremal chemical trees with respect to (reduced) Sombor index. It is worth noting that the relevant data of Table $1 \sim 8$ except the values of (reduced) Sombor indices are from $[15,17]$.

Table 1. $C T_{n}$ with $\Delta \leq 3, n_{3} \leq 2$ and their (reduced)Sombor index.

\begin{tabular}{c|ccccccc}
\hline & $m_{3,3}$ & $m_{2,3}$ & $m_{1,2}$ & $m_{1,3}$ & $m_{2,2}$ & $S O(G)$ & $S O_{\text {red }}(G)$ \\
\hline$A_{1}$ & 0 & 0 & 2 & 0 & $n-3$ & $(2 \sqrt{2} n-4.013145419)$ & $(\sqrt{2} n-2.242640687)$ \\
\hline$A_{2}$ & 0 & 1 & 1 & 2 & $n-5$ & $(2 \sqrt{2} n-1.975961050)$ & $(\sqrt{2} n+0.165000165)$ \\
\hline$A_{3}$ & 0 & 2 & 2 & 1 & $n-6$ & $(2 \sqrt{2} n-2.125046582)$ & $(\sqrt{2} n-0.013145419)$ \\
\hline$A_{4}$ & 0 & 3 & 3 & 0 & $n-7$ & $(2 \sqrt{2} n-2.274132114)$ & $(\sqrt{2} n-0.191291004)$ \\
\hline$A_{5}$ & 0 & 2 & 0 & 4 & $n-7$ & $(2 \sqrt{2} n+0.061223318)$ & $(\sqrt{2} n+2.572641018)$ \\
\hline$A_{6}$ & 0 & 3 & 1 & 3 & $n-8$ & $(2 \sqrt{2} n-0.087862213)$ & $(\sqrt{2} n+2.394495433)$ \\
\hline$A_{7}$ & 0 & 4 & 2 & 2 & $n-9$ & $(2 \sqrt{2} n-0.236947745)$ & $(\sqrt{2} n+2.216349848)$ \\
\hline$A_{8}$ & 1 & 1 & 1 & 3 & $n-7$ & $(2 \sqrt{2} n-0.227896952)$ & $(\sqrt{2} n+2.165000165)$ \\
\hline$A_{9}$ & 0 & 5 & 3 & 1 & $n-10$ & $(2 \sqrt{2} n-0.386033277)$ & $(\sqrt{2} n+2.038204263)$ \\
\hline$A_{10}$ & 1 & 2 & 2 & 2 & $n-8$ & $(2 \sqrt{2} n-0.376982484)$ & $(\sqrt{2} n+1.986854580)$ \\
\hline$A_{11}$ & 0 & 6 & 4 & 0 & $n-11$ & $(2 \sqrt{2} n-0.535118809)$ & $(\sqrt{2} n+1.860058678)$ \\
\hline$A_{12}$ & 1 & 3 & 3 & 1 & $n-9$ & $(2 \sqrt{2} n-0.526068016)$ & $(\sqrt{2} n+1.808708995)$ \\
\hline$A_{13}$ & 1 & 4 & 4 & 0 & $n-10$ & $(2 \sqrt{2} n-0.675153548)$ & $(\sqrt{2} n+1.630563411)$ \\
\hline
\end{tabular}

Theorem 3.3 If $n \geq 13, T_{1} \in A_{1}, T_{2} \in A_{4}, T_{3} \in A_{3}, T_{4} \in A_{2}, T_{5} \in A_{13}, T_{6} \in A_{11}$, $T_{7} \in A_{12}, T_{8} \in A_{9}, T_{9} \in A_{10}, T_{10} \in A_{7}, T_{11} \in A_{8}, T_{12} \in A_{6}, T_{13} \in A_{5}, T_{14} \in \Omega_{n}$, and 
$T \in C T_{n} \backslash\left\{T_{1}, T_{2}, \cdots, T_{14}\right\}$, then $S O\left(T_{1}\right)<S O\left(T_{2}\right)<S O\left(T_{3}\right)<S O\left(T_{4}\right)<S O\left(T_{5}\right)<$ $S O\left(T_{6}\right)<S O\left(T_{7}\right)<S O\left(T_{8}\right)<S O\left(T_{9}\right)<S O\left(T_{10}\right)<S O\left(T_{11}\right)<S O\left(T_{12}\right)<S O\left(T_{13}\right)<$ $S O\left(T_{14}\right)<S O(T)$.

Proof. By Table 1 and the Sombor index of chemical trees among $\Omega(n)$, we have $S O\left(T_{1}\right)<$ $S O\left(T_{2}\right)<S O\left(T_{3}\right)<S O\left(T_{4}\right)<S O\left(T_{5}\right)<S O\left(T_{6}\right)<S O\left(T_{7}\right)<S O\left(T_{8}\right)<S O\left(T_{9}\right)<$ $S O\left(T_{10}\right)<S O\left(T_{11}\right)<S O\left(T_{12}\right)<S O\left(T_{13}\right)<S O\left(T_{14}\right)$.

If $\Delta(T) \leq 3$ and $n_{3}(T) \leq 2$, the conclusion holds. If $\Delta(T)=3$ and $n_{3}(T) \geq 3$, then by Theorem 3.2, the conclusion holds. If $\Delta(T)=4$, then by Equation (1),(3) and Theorem 3.1 , the conclusion holds.

Similar to the proof of Theorem 3.3, we have

Theorem 3.4 If $n \geq 13, T_{1} \in A_{1}, T_{2} \in A_{4}, T_{3} \in A_{3}, T_{4} \in A_{2}, T_{5} \in A_{13}, T_{6} \in A_{12}, T_{7} \in$ $A_{11}, T_{8} \in A_{10}, T_{9} \in A_{9}, T_{10} \in A_{8}, T_{11} \in A_{7}, T_{12} \in A_{6}, T_{13} \in A_{5}, T_{14} \in \Omega_{n}$, and $T \in C T_{n} \backslash$ $\left\{T_{1}, T_{2}, \cdots, T_{14}\right\}$, then $S O_{\text {red }}\left(T_{1}\right)<S O_{\text {red }}\left(T_{2}\right)<S O_{\text {red }}\left(T_{3}\right)<S O_{\text {red }}\left(T_{4}\right)<S O_{\text {red }}\left(T_{5}\right)<$ $S O_{\text {red }}\left(T_{6}\right)<S O_{\text {red }}\left(T_{7}\right)<S O_{\text {red }}\left(T_{8}\right)<S O_{\text {red }}\left(T_{9}\right)<S O_{\text {red }}\left(T_{10}\right)<S O_{\text {red }}\left(T_{11}\right)<$ $S O_{\text {red }}\left(T_{12}\right)<S O_{\text {red }}\left(T_{13}\right)<S O_{\text {red }}\left(T_{14}\right)<S O_{\text {red }}(T)$.

\subsection{Chemical unicyclic graphs}

In this subsection, we consider the extremal chemical unicyclic graphs with respect to (reduced) Sombor index.

Table 2. Degree distributions $(D D)$ of $C U_{n}$ with $n_{1} \leq 2$.

\begin{tabular}{c|cccc}
\hline & $n_{4}$ & $n_{3}$ & $n_{2}$ & $n_{1}$ \\
\hline$H_{1}$ & 0 & 0 & $n$ & 0 \\
\hline$H_{2}$ & 0 & 1 & $n-2$ & 1 \\
\hline$H_{3}$ & 1 & 0 & $n-3$ & 2 \\
\hline$H_{4}$ & 0 & 2 & $n-4$ & 2 \\
\hline
\end{tabular}

Lemma 3.5 $[15,17] G \in C U_{n}$ and $n_{1}(G) \leq 2$ if and only if $G$ belongs to one of equivalence classes given in Table 2.

Theorem 3.6 If $n \geq 7, G_{1} \in \alpha_{1}, G_{2} \in \alpha_{3}, G_{3} \in \alpha_{2}, G_{4} \in \alpha_{9}$ in Table 3. $G \in$ $C U_{n} \backslash\left\{G_{1}, G_{2}, G_{3}, G_{4}\right\}$, then $S O\left(G_{1}\right)<S O\left(G_{2}\right)<S O\left(G_{3}\right)<S O\left(G_{4}\right)<S O(G)$.

Proof. By Table 3, we have $S O\left(G_{1}\right)<S O\left(G_{2}\right)<S O\left(G_{3}\right)<S O\left(G_{4}\right)$. 
Table 3. $C U_{n}$ with $n_{1} \leq 2$ and their (reduced)Sombor index.

\begin{tabular}{c|cccccccccc}
\hline & $D D$ & $m_{1,2}$ & $m_{1,3}$ & $m_{1,4}$ & $m_{2,3}$ & $m_{2,4}$ & $m_{3,3}$ & $m_{2,2}$ & $S O(G)$ & $S O_{\text {red }}(G)$ \\
\hline$\alpha_{1}$ & $H_{1}$ & 0 & 0 & 0 & 0 & 0 & 0 & $n$ & $(2 \sqrt{2} n)$ & $(\sqrt{2} n)$ \\
\hline$\alpha_{2}$ & $H_{2}$ & 0 & 1 & 0 & 2 & 0 & 0 & $n-3$ & $(2 \sqrt{2} n+1.888)$ & $(\sqrt{2} n+2.229)$ \\
\hline$\alpha_{3}$ & $H_{2}$ & 1 & 0 & 0 & 3 & 0 & 0 & $n-4$ & $(2 \sqrt{2} n+1.739)$ & $(\sqrt{2} n+2.051)$ \\
\hline$\alpha_{4}$ & $H_{3}$ & 0 & 0 & 2 & 0 & 2 & 0 & $n-4$ & $(2 \sqrt{2} n+5.876)$ & $(\sqrt{2} n+6.667)$ \\
\hline$\alpha_{5}$ & $H_{3}$ & 1 & 0 & 1 & 0 & 3 & 0 & $n-5(2 \sqrt{2} n+5.633)$ & $(\sqrt{2} n+6.415)$ \\
\hline$\alpha_{6}$ & $H_{3}$ & 2 & 0 & 0 & 0 & 4 & 0 & $n-6(2 \sqrt{2} n+5.390)$ & $(\sqrt{2} n+6.163)$ \\
\hline$\alpha_{7}$ & $H_{4}$ & 0 & 2 & 0 & 2 & 0 & 1 & $n-5(2 \sqrt{2} n+3.636)$ & $(\sqrt{2} n+4.229)$ \\
\hline$\alpha_{8}$ & $H_{4}$ & 1 & 1 & 0 & 3 & 0 & 1 & $n-6(2 \sqrt{2} n+3.487)$ & $(\sqrt{2} n+4.051)$ \\
\hline$\alpha_{9}$ & $H_{4}$ & 2 & 0 & 0 & 4 & 0 & 1 & $n-7(2 \sqrt{2} n+3.337)$ & $(\sqrt{2} n+3.873)$ \\
\hline$\alpha_{10}$ & $H_{4}$ & 0 & 2 & 0 & 4 & 0 & 0 & $n-6(2 \sqrt{2} n+3.776)$ & $(\sqrt{2} n+4.458)$ \\
\hline$\alpha_{11}$ & $H_{4}$ & 1 & 1 & 0 & 5 & 0 & 0 & $n-7(2 \sqrt{2} n+3.627)$ & $(\sqrt{2} n+4.280)$ \\
\hline$\alpha_{12}$ & $H_{4}$ & 2 & 0 & 0 & 6 & 0 & 0 & $n-8(2 \sqrt{2} n+3.478)$ & $(\sqrt{2} n+4.102)$ \\
\hline
\end{tabular}

If $n_{1}(G) \leq 2$, by Table 3 , the conclusion holds. If $n_{1}(G) \geq 3$, by the transformations of Lemma 2.3 and Lemma 2.4, we can obtain a chemical unicyclic graphs $G^{*}$ with $n_{1}\left(G^{*}\right)=2$, so we have $S O(G)>S O\left(G^{*}\right)$. By Table 3, $S O\left(G_{4}\right) \leq S O\left(G^{*}\right)$. Thus, the conclusion holds.

Similar to the proof of Theorem 3.6, we have

Theorem 3.7 If $n \geq 7, G_{1} \in \alpha_{1}, G_{2} \in \alpha_{3}, G_{3} \in \alpha_{2}, G_{4} \in \alpha_{9}$ in Table 3. $G \in C U_{n} \backslash$ $\left\{G_{1}, G_{2}, G_{3}, G_{4}\right\}$, then $S O_{\text {red }}\left(G_{1}\right)<S O_{\text {red }}\left(G_{2}\right)<S O_{\text {red }}\left(G_{3}\right)<S O_{\text {red }}\left(G_{4}\right)<S O_{\text {red }}(G)$.

\subsection{Chemical bicyclic graphs}

In this subsection, we consider the extremal chemical bicyclic graphs with respect to (reduced) Sombor index.

Table 4. Degree distributions $(D D)$ of $C B_{n}$ with $n_{1} \leq 1$.

\begin{tabular}{c|cccc}
\hline & $n_{4}$ & $n_{3}$ & $n_{2}$ & $n_{1}$ \\
\hline$B_{1}$ & 1 & 0 & $n-1$ & 0 \\
\hline$B_{2}$ & 0 & 2 & $n-2$ & 0 \\
\hline$B_{3}$ & 1 & 1 & $n-3$ & 1 \\
\hline$B_{4}$ & 0 & 3 & $n-4$ & 1 \\
\hline
\end{tabular}

Lemma $3.8[15,17] G \in C B_{n}$ and $n_{1}(G) \leq 1$ if and only if $G$ belongs to one of equivalence classes given in Table 4 .

Theorem 3.9 If $n \geq 6, G_{1} \in \beta_{2}, G_{2} \in \beta_{3}, G_{3} \in \beta_{9}$ in Table 5. $G \in C B_{n} \backslash\left\{G_{1}, G_{2}, G_{3}\right\}$, then $S O\left(G_{1}\right)<S O\left(G_{2}\right)<S O\left(G_{3}\right)<S O(G)$. 
Table 5. $C B_{n}$ with $n_{1} \leq 1$ and their (reduced)Sombor index.

\begin{tabular}{c|cccccccccccc}
\hline & $D D$ & $m_{1,2}$ & $m_{1,3}$ & $m_{1,4}$ & $m_{2,3}$ & $m_{2,4}$ & $m_{3,3}$ & $m_{3,4}$ & $m_{2,2}$ & $S O(G)$ & $S O_{\text {red }}(G)$ \\
\hline$\beta_{1}$ & $B_{1}$ & 0 & 0 & 0 & 0 & 4 & 0 & 0 & $n-3$ & $(2 \sqrt{2} n+9.403)$ & $(\sqrt{2} n+8.406)$ \\
\hline$\beta_{2}$ & $B_{2}$ & 0 & 0 & 0 & 4 & 0 & 1 & 0 & $n-4$ & $(2 \sqrt{2} n+7.351)$ & $(\sqrt{2} n+6.115)$ \\
\hline$\beta_{3}$ & $B_{2}$ & 0 & 0 & 0 & 6 & 0 & 0 & 0 & $n-5$ & $(2 \sqrt{2} n+7.491)$ & $(\sqrt{2} n+6.345)$ \\
\hline$\beta_{4}$ & $B_{3}$ & 0 & 0 & 1 & 2 & 2 & 0 & 1 & $n-5$ & $(2 \sqrt{2} n+11.136)$ & $(\sqrt{2} n+10.331)$ \\
\hline$\beta_{5}$ & $B_{3}$ & 1 & 0 & 0 & 2 & 3 & 0 & 1 & $n-6$ & $(2 \sqrt{2} n+10.893)$ & $(\sqrt{2} n+10.079)$ \\
\hline$\beta_{6}$ & $B_{3}$ & 0 & 0 & 1 & 3 & 3 & 0 & 0 & $n-6$ & $(2 \sqrt{2} n+11.385)$ & $(\sqrt{2} n+10.709)$ \\
\hline$\beta_{7}$ & $B_{3}$ & 1 & 0 & 0 & 3 & 4 & 0 & 0 & $n-7$ & $(2 \sqrt{2} n+11.142)$ & $(\sqrt{2} n+10.457)$ \\
\hline$\beta_{8}$ & $B_{4}$ & 0 & 1 & 0 & 2 & 0 & 3 & 0 & $n-5$ & $(2 \sqrt{2} n+8.959)$ & $(\sqrt{2} n+7.886)$ \\
\hline$\beta_{9}$ & $B_{4}$ & 1 & 0 & 0 & 3 & 0 & 3 & 0 & $n-6$ & $(2 \sqrt{2} n+8.810)$ & $(\sqrt{2} n+7.708)$ \\
\hline$\beta_{10}$ & $B_{4}$ & 0 & 1 & 0 & 4 & 0 & 2 & 0 & $n-6$ & $(2 \sqrt{2} n+9.099)$ & $(\sqrt{2} n+8.115)$ \\
\hline$\beta_{11}$ & $B_{4}$ & 1 & 0 & 0 & 5 & 0 & 2 & 0 & $n-7$ & $(2 \sqrt{2} n+8.950)$ & $(\sqrt{2} n+7.937)$ \\
\hline$\beta_{12}$ & $B_{4}$ & 0 & 1 & 0 & 6 & 0 & 1 & 0 & $n-7$ & $(2 \sqrt{2} n+9.239)$ & $(\sqrt{2} n+8.345)$ \\
\hline$\beta_{13}$ & $B_{4}$ & 1 & 0 & 0 & 7 & 0 & 1 & 0 & $n-8$ & $(2 \sqrt{2} n+9.090)$ & $(\sqrt{2} n+8.167)$ \\
\hline$\beta_{14}$ & $B_{4}$ & 0 & 1 & 0 & 8 & 0 & 0 & 0 & $n-8$ & $(2 \sqrt{2} n+9.379)$ & $(\sqrt{2} n+8.574)$ \\
\hline$\beta_{15}$ & $B_{4}$ & 1 & 0 & 0 & 9 & 0 & 0 & 0 & $n-9$ & $(2 \sqrt{2} n+9.230)$ & $(\sqrt{2} n+8.396)$ \\
\hline
\end{tabular}

Proof. By Table 5, we have $S O\left(G_{1}\right)<S O\left(G_{2}\right)<S O\left(G_{3}\right)$.

If $n_{1}(G) \leq 1$, by Table 5 , the conclusion holds. If $n_{1}(G) \geq 2$, by the transformations of Lemma 2.3 and Lemma 2.4, we can obtain a chemical bicyclic graphs $G^{*}$ with $n_{1}\left(G^{*}\right)=1$, so we have $S O(G)>S O\left(G^{*}\right)$. By Table $5, S O\left(G_{3}\right) \leq S O\left(G^{*}\right)$. Thus, the conclusion holds.

Similar to the proof of Theorem 3.9, we have

Theorem 3.10 If $n \geq 6, G_{1} \in \beta_{2}, G_{2} \in \beta_{3}, G_{3} \in \beta_{9}$ in Table 5. $G \in C B_{n} \backslash\left\{G_{1}, G_{2}, G_{3}\right\}$, then $S O_{\text {red }}\left(G_{1}\right)<S O_{\text {red }}\left(G_{2}\right)<S O_{\text {red }}\left(G_{3}\right)<S O_{\text {red }}(G)$.

\subsection{Chemical tricyclic graphs}

In this subsection, we consider the extremal chemical tricyclic graphs with respect to (reduced) Sombor index.

Table 6. Degree distributions $(D D)$ of $C T G_{n}$ with $n_{1} \leq 1$.

\begin{tabular}{c|cccc}
\hline & $n_{4}$ & $n_{3}$ & $n_{2}$ & $n_{1}$ \\
\hline$E_{1}$ & 2 & 0 & $n-2$ & 0 \\
\hline$E_{2}$ & 1 & 2 & $n-3$ & 0 \\
\hline$E_{3}$ & 0 & 4 & $n-4$ & 0 \\
\hline$E_{4}$ & 2 & 1 & $n-4$ & 1 \\
\hline$E_{5}$ & 1 & 3 & $n-5$ & 1 \\
\hline$E_{6}$ & 0 & 5 & $n-6$ & 1 \\
\hline
\end{tabular}


Table 7. $C T G_{n}$ with $n_{1} \leq 1$ and their (reduced)Sombor index.

\begin{tabular}{|c|c|c|c|c|c|c|c|c|c|c|}
\hline & $D D$ & & & & & & & & $n_{4,4}$ & $S O_{\text {red }}(G)$ \\
\hline$\gamma_{1}$ & $E_{1}$ & 0 & 0 & 0 & 0 & 8 & 0 & 0 & 0 & $n-6(2 \sqrt{2} n+18.806)(\sqrt{2} n+16.812)$ \\
\hline$\gamma_{2}$ & $E_{1}$ & 0 & 0 & 0 & 0 & 6 & 0 & 0 & 1 & $n-5(2 \sqrt{2} n+18.347)(\sqrt{2} n+16.145)$ \\
\hline$\gamma_{3}$ & $E_{2}$ & 0 & 0 & 0 & 2 & 2 & 1 & 2 & 0 & $n-5(2 \sqrt{2} n+16.255)(\sqrt{2} n+13.765)$ \\
\hline$\gamma_{4}$ & $E_{2}$ & 0 & 0 & 0 & 3 & 3 & 1 & 1 & 0 & $n-6(2 \sqrt{2} n+16.505)(\sqrt{2} n+14.143)$ \\
\hline$\gamma_{5}$ & $E_{2}$ & 0 & 0 & 0 & 4 & 4 & & & 0 & $n-7(2 \sqrt{2} n+16.754)(\sqrt{2} n+14.522)$ \\
\hline$\gamma_{6}$ & $E_{2}$ & 0 & 0 & 0 & 4 & 2 & 0 & 2 & 0 & $n-6(2 \sqrt{2} n+16.395)(\sqrt{2} n+13.994)$ \\
\hline$\gamma_{7}$ & $E_{2}$ & 0 & 0 & 0 & 5 & 3 & 0 & 1 & 0 & $n-7(2 \sqrt{2} n+16.645)(\sqrt{2} n+14.373)$ \\
\hline$\gamma_{8}$ & $E_{2}$ & 0 & 0 & 0 & 6 & 4 & U & 0 & 0 & $n-8(2 \sqrt{2} n+16.894)(\sqrt{2} n+14.751)$ \\
\hline$\gamma_{9}$ & $E_{3}$ & 0 & 0 & 0 & 2 & 0 & 5 & 0 & 0 & $n-5(2 \sqrt{2} n+14.282)(\sqrt{2} n+11.543)$ \\
\hline$\gamma_{10}$ & $E_{3}$ & 0 & 0 & 0 & 4 & 0 & 4 & 0 & 0 & $n-6(2 \sqrt{2} n+14.422)(\sqrt{2} n+11.772)$ \\
\hline$\gamma_{11}$ & $E_{3}$ & 0 & 0 & 0 & 6 & 0 & 3 & 0 & 0 & $n-7(2 \sqrt{2} n+14.562)(\sqrt{2} n+12.002)$ \\
\hline$\gamma_{12}$ & $E_{3}$ & 0 & 0 & 0 & 8 & 0 & 2 & ( & 0 & $n-8(2 \sqrt{2} n+14.702)(\sqrt{2} n+12.231)$ \\
\hline$\gamma_{13}$ & $E_{3}$ & 0 & 0 & 0 & 10 & 0 & 1 & 0 & 0 & $n-9(2 \sqrt{2} n+14.842)(\sqrt{2} n+12.461)$ \\
\hline$\gamma_{14}$ & $E_{3}$ & 0 & 0 & 0 & 12 & 0 & 0 & 0 & 0 & $n-10(2 \sqrt{2} n+14.982)(\sqrt{2} n+12.690)$ \\
\hline$\gamma_{15}$ & $E_{4}$ & 0 & 0 & 1 & 1 & 3 & 0 & & 1 & $n-6(2 \sqrt{2} n+19.831)(\sqrt{2} n+17.691)$ \\
\hline$\gamma_{16}$ & $E_{4}$ & 1 & 0 & 0 & 1 & 4 & 0 & 2 & 1 & $n-7(2 \sqrt{2} n+19.588)(\sqrt{2} n+17.439)$ \\
\hline$\gamma_{17}$ & $E_{4}$ & 0 & 0 & 1 & 2 & 4 & 0 & 1 & 1 & $n-7(2 \sqrt{2} n+20.080)(\sqrt{2} n+18.069)$ \\
\hline$\gamma_{18}$ & $E_{4}$ & 1 & 0 & 0 & 2 & 5 & 0 & 1 & 1 & $n-8(2 \sqrt{2} n+19.837)(\sqrt{2} n+17.818)$ \\
\hline$\gamma_{19}$ & $E_{4}$ & 0 & 0 & 1 & 3 & 5 & 0 & ( & 1 & $n-8(2 \sqrt{2} n+20.329)(\sqrt{2} n+18.448)$ \\
\hline$\gamma_{20}$ & $E_{4}$ & 1 & 0 & 0 & 3 & 6 & 0 & 0 & 1 & $n-9(2 \sqrt{2} n+20.086)(\sqrt{2} n+18.196)$ \\
\hline$\gamma_{21}$ & $E_{4}$ & 0 & 0 & 1 & 1 & 5 & 0 & 2 & 0 & $n-7(2 \sqrt{2} n+20.290)(\sqrt{2} n+18.359)$ \\
\hline$\gamma_{22}$ & $E_{4}$ & 1 & 0 & 0 & 1 & 6 & U & & 0 & $n-8(2 \sqrt{2} n+20.047)(\sqrt{2} n+18.107)$ \\
\hline$\gamma_{23}$ & $E_{4}$ & 0 & 0 & 1 & 2 & 6 & 0 & 1 & 0 & $n-8(2 \sqrt{2} n+20.539)(\sqrt{2} n+18.737)$ \\
\hline$\gamma_{24}$ & $E_{4}$ & 1 & 0 & 0 & 2 & 7 & 0 & 1 & 0 & $n-9(2 \sqrt{2} n+20.296)(\sqrt{2} n+18.485)$ \\
\hline$\gamma_{25}$ & $E_{4}$ & 0 & 0 & 1 & 3 & 7 & U & 0 & 0 & $n-9(2 \sqrt{2} n+20.788)(\sqrt{2} n+16.116)$ \\
\hline$\gamma_{26}$ & $E_{4}$ & 1 & 0 & 0 & 3 & 8 & 0 & 0 & 0 & $n-10(2 \sqrt{2} n+20.545)(\sqrt{2} n+18.864)$ \\
\hline$\gamma_{27}$ & $E_{5}$ & 0 & 0 & 1 & 2 & 0 & ? & 3 & 0 & $n-6(2 \sqrt{2} n+17.848)(\sqrt{2} n+15.460)$ \\
\hline$\gamma_{28}$ & $E_{5}$ & 0 & 0 & 1 & 4 & 0 & 1 & 3 & 0 & $n-7(2 \sqrt{2} n+17.988)(\sqrt{2} n+15.689)$ \\
\hline$\gamma_{29}$ & $E_{5}$ & 0 & 0 & 1 & 6 & 0 & 0 & 3 & 0 & $n-8(2 \sqrt{2} n+18.128)(\sqrt{2} n+15.919)$ \\
\hline$\gamma_{30}$ & $E_{5}$ & 0 & 0 & 1 & 1 & 1 & 3 & 2 & 0 & $n-6(2 \sqrt{2} n+17.958)(\sqrt{2} n+15.609)$ \\
\hline$\gamma_{31}$ & $E_{5}$ & 0 & 0 & 1 & 3 & 1 & 2 & 2 & 0 & $n-7(2 \sqrt{2} n+18.098)(\sqrt{2} n+15.838)$ \\
\hline$\gamma_{32}$ & $E_{5}$ & 0 & 0 & 1 & 5 & 1 & 1 & 2 & 0 & $n-8(2 \sqrt{2} n+18.238)(\sqrt{2} n+16.068)$ \\
\hline$\gamma_{33}$ & $E_{5}$ & 0 & 0 & 1 & 7 & 1 & 0 & 2 & 0 & $n-9(2 \sqrt{2} n+18.378)(\sqrt{2} n+16.297)$ \\
\hline$\gamma_{34}$ & $E_{5}$ & 0 & 0 & 1 & 2 & 2 & 3 & 1 & 0 & $n-7(2 \sqrt{2} n+18.207)(\sqrt{2} n+15.988)$ \\
\hline$\gamma_{35}$ & $E_{5}$ & 0 & 0 & 1 & 4 & 2 & 2 & 1 & 0 & $n-8(2 \sqrt{2} n+18.347)(\sqrt{2} n+16.217)$ \\
\hline$\gamma_{36}$ & $E_{5}$ & 0 & 0 & 1 & 6 & 2 & 1 & 1 & 0 & $n-9(2 \sqrt{2} n+18.487)(\sqrt{2} n+16.447)$ \\
\hline$\gamma_{37}$ & $E_{5}$ & 0 & 0 & 1 & 8 & 2 & U & 1 & 0 & $n-10(2 \sqrt{2} n+18.627)(\sqrt{2} n+16.676)$ \\
\hline$\gamma_{38}$ & $E_{5}$ & 0 & 0 & 1 & 3 & 3 & 3 & 0 & 0 & $n-8(2 \sqrt{2} n+18.456)(\sqrt{2} n+16.366)$ \\
\hline$\gamma_{39}$ & $E_{5}$ & 0 & 0 & 1 & 5 & 3 & 2 & 0 & 0 & $n-9(2 \sqrt{2} n+18.596)(\sqrt{2} n+16.596)$ \\
\hline$\gamma_{40}$ & $E_{5}$ & 0 & 0 & 1 & 7 & 3 & 1 & 0 & 0 & $n-10(2 \sqrt{2} n+18.736)(\sqrt{2} n+16.825)$ \\
\hline
\end{tabular}


Table 8. $C T G_{n}$ with $n_{1} \leq 1$ and their (reduced)Sombor index.

\begin{tabular}{|c|c|c|c|c|c|c|c|c|c|c|}
\hline & $D D$ & & & & & & & & $n_{4,4}$ & $\begin{array}{lll}m_{2,2} & S O(G) & S O_{\text {red }}(G)\end{array}$ \\
\hline$\gamma_{41}$ & $E_{5}$ & 0 & 0 & 1 & 9 & 3 & 0 & 0 & 0 & $n-11(2 \sqrt{2} n+18.876)(\sqrt{2} n+17.055)$ \\
\hline$\gamma_{42}$ & $E_{5}$ & 1 & U & 0 & 0 & 1 & 3 & 3 & 0 & $n-6(2 \sqrt{2} n+17.465)(\sqrt{2} n+14.978)$ \\
\hline$\gamma_{43}$ & $E_{5}$ & 1 & U & 0 & 2 & 1 & 2 & 3 & 0 & $n-7(2 \sqrt{2} n+17.605)(\sqrt{2} n+15.208)$ \\
\hline$\gamma_{44}$ & $E_{5}$ & 1 & & 0 & 4 & & & 3 & 0 & $n-8(2 \sqrt{2} n+17.745)(\sqrt{2} n+15.437)$ \\
\hline & $E_{5}$ & 1 & & 0 & 6 & 1 & & 2 & 0 & $n-9(2 \sqrt{2} n+17.885)(\sqrt{2} n+15.667)$ \\
\hline$\gamma_{46}$ & $E_{5}$ & 1 & U & 0 & 1 & 2 & 3 & 2 & 0 & $n-7(2 \sqrt{2} n+17.714)(\sqrt{2} n+15.357)$ \\
\hline$\gamma_{47}$ & $E_{5}$ & 1 & & 0 & 3 & & 2 & 2 & 0 & $n-8(2 \sqrt{2} n+17.854)(\sqrt{2} n+15.587)$ \\
\hline & $E_{5}$ & 1 & ( & 0 & 5 & 2 & & 2 & 0 & $n-9(2 \sqrt{2} n+17.994)(\sqrt{2} n+15.816)$ \\
\hline$\gamma_{49}$ & $E_{5}$ & 1 & 0 & 0 & 7 & 2 & 0 & 2 & 0 & $n-10(2 \sqrt{2} n+18.134)(\sqrt{2} n+16.045)$ \\
\hline$\gamma_{50}$ & $E_{5}$ & 1 & 0 & 0 & 2 & 3 & 3 & 1 & 0 & $n-8(2 \sqrt{2} n+17.964)(\sqrt{2} n+15.736)$ \\
\hline$\gamma_{51}$ & $E_{5}$ & 1 & & 0 & 4 & 3 & 2 & 1 & 1 & $n-9(2 \sqrt{2} n+18.104)(\sqrt{2} n+15.965)$ \\
\hline$\gamma_{52}$ & $E_{5}$ & 1 & & 0 & 6 & 3 & & & 0 & $n-10(2 \sqrt{2} n+18.244)(\sqrt{2} n+16.195)$ \\
\hline$\gamma_{53}$ & $E_{5}$ & 1 & 0 & 0 & 8 & 3 & 0 & 1 & 0 & $n-11(2 \sqrt{2} n+18.384)(\sqrt{2} n+16.424)$ \\
\hline$\gamma_{54}$ & $E_{5}$ & 1 & 0 & 0 & 3 & 4 & 3 & 0 & 0 & $n-9(2 \sqrt{2} n+18.213)(\sqrt{2} n+16.114)$ \\
\hline$\gamma_{55}$ & $E_{5}$ & 1 & & 0 & 5 & 4 & & 0 & 0 & $n-10(2 \sqrt{2} n+18.353)(\sqrt{2} n+16.344)$ \\
\hline$\gamma_{56}$ & $E_{5}$ & 1 & 0 & 0 & 7 & 4 & 1 & 0 & 0 & $n-11(2 \sqrt{2} n+18.493)(\sqrt{2} n+16.573)$ \\
\hline 57 & $E_{5}$ & 1 & 0 & 0 & 9 & 4 & 0 & 0 & 0 & $n-12(2 \sqrt{2} n+18.633)(\sqrt{2} n+16.803)$ \\
\hline$\gamma_{58}$ & $E_{6}$ & 0 & I & 0 & 2 & 0 & & 0 & 0 & $n-7(2 \sqrt{2} n+16.030)(\sqrt{2} n+13.543)$ \\
\hline$\gamma_{59}$ & $E_{6}$ & 0 & 1 & 0 & 4 & 0 & & 0 & 0 & $n-8(2 \sqrt{2} n+16.170)(\sqrt{2} n+13.772)$ \\
\hline & $E_{6}$ & 0 & 1 & 0 & 6 & 0 & 4 & 0 & 0 & $n-9(2 \sqrt{2} n+16.310)(\sqrt{2} n+14.002)$ \\
\hline & $E_{6}$ & 0 & 1 & 0 & 8 & 0 & 3 & 0 & 0 & $n-10(2 \sqrt{2} n+16.450)(\sqrt{2} n+14.231)$ \\
\hline$\gamma_{62}$ & $E_{6}$ & 0 & 1 & 0 & 10 & 0 & 2 & 0 & 0 & $n-11(2 \sqrt{2} n+16.590)(\sqrt{2} n+14.461)$ \\
\hline 863 & $E_{6}$ & 0 & & 0 & 12 & 0 & 1 & 0 & 0 & $n-12(2 \sqrt{2} n+16.730)(\sqrt{2} n+14.690)$ \\
\hline & $E_{6}$ & 0 & 1 & 0 & 14 & 0 & 0 & 0 & 0 & $n-13(2 \sqrt{2} n+16.870)(\sqrt{2} n+14.920)$ \\
\hline & $E_{6}$ & 1 & 0 & 0 & 1 & 0 & 7 & 0 & 0 & $n-7(2 \sqrt{2} n+15.741)(\sqrt{2} n+13.135)$ \\
\hline 66 & $E_{6}$ & 1 & & 0 & 3 & 0 & 0 & 0 & 0 & $n-8(2 \sqrt{2} n+15.881)(\sqrt{2} n+13.365)$ \\
\hline & $E_{6}$ & 1 & 0 & 0 & 5 & 0 & & 0 & 0 & $n-9(2 \sqrt{2} n+16.021)(\sqrt{2} n+13.594)$ \\
\hline$\gamma_{68}$ & $E_{6}$ & 1 & 0 & 0 & 7 & 0 & 4 & 0 & 0 & $n-10(2 \sqrt{2} n+16.161)(\sqrt{2} n+13.824)$ \\
\hline & $E_{6}$ & 1 & 0 & 0 & 9 & 0 & 3 & 0 & 0 & $n-11(2 \sqrt{2} n+16.301)(\sqrt{2} n+14.053)$ \\
\hline$\gamma_{70}$ & $E_{6}$ & 1 & 0 & 0 & 11 & 0 & & 0 & 0 & $n-12(2 \sqrt{2} n+16.441)(\sqrt{2} n+14.283)$ \\
\hline$\gamma_{71}$ & $E_{6}$ & 1 & 0 & 0 & 13 & 0 & & 0 & 0 & $n-13(2 \sqrt{2} n+16.581)(\sqrt{2} n+14.512)$ \\
\hline$\gamma_{72}$ & $E_{6}$ & 1 & 0 & 0 & 15 & 0 & 0 & 0 & 0 & $n-14(2 \sqrt{2} n+16.721)(\sqrt{2} n+14.742)$ \\
\hline
\end{tabular}

Lemma $3.11[15,17] G \in C T G_{n}$ and $n_{1}(G) \leq 1$ if and only if $G$ belongs to one of equivalence classes given in Table 6 .

Theorem 3.12 If $n \geq 6, G_{1} \in \gamma_{9}, G_{2} \in \gamma_{10}, G_{3} \in \gamma_{11}, G_{4} \in \gamma_{12}, G_{5} \in \gamma_{13}, G_{6} \in$ $\gamma_{14}, G_{7} \in \gamma_{65}$ in Table 7 and Table 8. $G \in C T G_{n} \backslash\left\{G_{1}, G_{2}, G_{3}, G_{4}, G_{5}, G_{6}, G_{7}\right\}$, then $S O\left(G_{1}\right)<S O\left(G_{2}\right)<S O\left(G_{3}\right)<S O\left(G_{4}\right)<S O\left(G_{5}\right)<S O\left(G_{6}\right)<S O\left(G_{7}\right)<S O(G)$.

Proof. By Table 7 and Table 8, we have $S O\left(G_{1}\right)<S O\left(G_{2}\right)<S O\left(G_{3}\right)<S O\left(G_{4}\right)<$ 
$S O\left(G_{5}\right)<S O\left(G_{6}\right)<S O\left(G_{7}\right)$.

If $n_{1}(G) \leq 1$, by Table 7 and Table 8 , the conclusion holds. If $n_{1}(G) \geq 2$, by the transformations of Lemma 2.3 and Lemma 2.4, we can obtain a chemical tricyclic graphs $G^{*}$ with $n_{1}\left(G^{*}\right)=1$, so we have $S O(G)>S O\left(G^{*}\right)$. By Table 7 and Table 8 , $S O\left(G_{7}\right) \leq S O\left(G^{*}\right)$. Thus, the conclusion holds.

Similar to the proof of Theorem 3.12, we have

Theorem 3.13 If $n \geq 6, G_{1} \in \gamma_{9}, G_{2} \in \gamma_{10}, G_{3} \in \gamma_{11}, G_{4} \in \gamma_{12}, G_{5} \in \gamma_{13}, G_{6} \in \gamma_{14}$, $G_{7} \in \gamma_{65}$ in Table 7 and Table 8. $G \in C T G_{n} \backslash\left\{G_{1}, G_{2}, G_{3}, G_{4}, G_{5}, G_{6}, G_{7}\right\}$, then $S O_{\text {red }}\left(G_{1}\right)<S O_{\text {red }}\left(G_{2}\right)<S O_{\text {red }}\left(G_{3}\right)<S O_{\text {red }}\left(G_{4}\right)<S O_{\text {red }}\left(G_{5}\right)<S O_{\text {red }}\left(G_{6}\right)<$ $S O_{\text {red }}\left(G_{7}\right)<S O_{\text {red }}(G)$.

\section{Applications of reduced Sombor index to octane isomers}

Deng et al. [9], considered the correlation between some physico-chemical properties of octane isomers with Sombor index. In this section, we study the correlation between these physico-chemical properties of octane isomers with reduced Sombor index. We also compare the reduced Sombor index with some other topological indices.

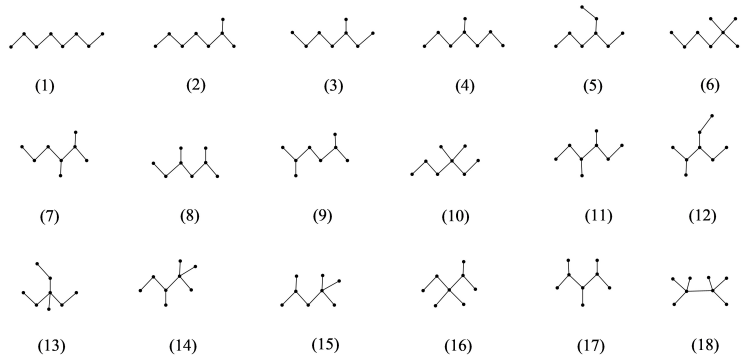

Figure 1. Chemical graphs of octane isomers.

The chemical graphs of 18 octane isomers can see in Figure 1. We can calculate the values of reduced Sombor index for the 18 octane isomers in Figure 1 as [9.0710, 11.4787, 11.30 05, 11.3005, 11.1224, 15.9907, 13.4787, 13.7082, 13.8663, 15.7387, 13.3005, 13.3005, 15.4868, 17.8416, 18.3983, 17.7678, 15.6568, 22.2426]. Based on the values of Acentric Factors (Entropy, SNar, HNar) of the 18 octane isomers (see $[7,9]$ ) in Figure 1, we can also obtain 
the regression models of the reduces Sombor index (similar to the results of [9]).

$$
\begin{gathered}
\text { AcenFac }=0.4881-0.0105 \times S O_{\text {red }}, R^{2}=0.9213 . \\
\text { Entropy }=124.5-1.317 \times S O_{r e d}, R^{2}=0.8922 . \\
S N a r=5.003-0.1015 \times S O_{r e d}, R^{2}=0.9736 \\
H N a r=1.793-0.02654 \times S O_{r e d}, R^{2}=0.9341
\end{gathered}
$$

Table 9. $R^{2}$ values between indices and Acentric Factors, Entropy, SNar, HNar.

\begin{tabular}{c|cccccccc}
\hline Physico-chemical property & $S O_{\text {red }}$ & $M_{1}$ & $M_{2}$ & $F$ & $R$ & $S C I$ & $S D D$ & $M_{N}$ \\
\hline Acentric Factors & 0.9213 & 0.9468 & 0.973 & 0.9313 & 0.8176 & 0.8647 & 0.8118 & 0.98915 \\
\hline Entropy & 0.8922 & 0.9107 & 0.8868 & 0.9077 & 0.8205 & 0.8518 & 0.8276 & 0.90746 \\
\hline SNar & 0.9736 & 0.9974 & 0.8940 & 0.9453 & 0.9487 & 0.9710 & 0.9252 & 0.9477 \\
\hline HNar & 0.9341 & 0.9774 & 0.8941 & 0.9453 & 0.9487 & 0.9710 & 0.9252 & 0.9115 \\
\hline
\end{tabular}

The correlation $(R)$ between Acentric Factors(resp. Entropy, SNar, HNar) and reduced Sombor indices of the octane isomers is about -0.959 (resp. $-0.944,-0.986,-0.966$ ). It shows a good linear relation. Therefore, the reduced Sombor index can help to predict these physico-chemical properties. We compare the reduced Sombor index with some existing topological indices, we found that sometimes the reduced Sombor index shows better predictive power than the existing indices. It is worth noting that [24] (before our paper) also consider the correlation between Sombor index and Entropy of octane isomers, however, for the sake of the integrity of the article, we have not removed the results about our results of Entropy. We also considered other physico-chemical properties, such as Acentric Factors, SNar and HNar, which do not appear in the [24].

\section{Concluding Remarks}

In this paper, we determine the first fourteen minimum chemical trees, the first four minimum chemical unicyclic graphs, the first three minimum chemical bicyclic graphs, the first seven minimum chemical tricyclic graphs. At last, we consider applications of reduced Sombor index to octane isomers. However, obtaining a more detailed ordering is still an open problem.

Problem 5.1 Further ordering chemical graphs by their Sombor indices. 
We intend to elaborate this matter in the near future.

Acknowledgment: The authors gratefully thank the anonymous referees for the constructive comments and recommendations which help to improve the readability and quality of the paper. Liu was supported by the Hunan Provincial Natural Science Foundation of China (Grant No. 2020JJ4423) and the Department of Education of Hunan Province (Grant No. 19A318). You was supported by the National Natural Science Foundation of China (Grant No.11971180) and the Guangdong Provincial Natural Science Foundation (Grant No. 2019A1515012052). Huang was supported by the National Natural Science Foundation of China (Grant No.11501139), the characteristic innovation project of general colleges and universities in Guangdong Province (Grant No. 2019GKTSCX001) and the Key Project at School Level of Guangzhou Civil Aviation College (Grant No. 18X0429).

\section{References}

[1] A. Ali, Z. Du, M. Ali, A note on chemical trees with minimum Wiener polarity index, Appl. Math. Comput. 335 (2018) 231-236.

[2] S. Alikhani, N. Ghanbari, Sombor index of polymers, MATCH Commun. Math. Comput. Chem. 86 (2021) 715-728.

[3] A. R. Ashrafi, A. Ghalavand, Ordering chemical trees by Wiener polarity index, Appl. Math. Comput. 313 (2017) 301-312.

[4] J. A. Bondy, U. S. R. Murty, Graph Theory, Springer, New York, 2008.

[5] R. Cruz, I. Gutman, J. Rada, Sombor index of chemical graphs, Appl. Math. Comput. 399 (2021) \#126018.

[6] R. Cruz, J. Rada, Extremal values of the Sombor index in unicyclic and bicyclic graphs, J. Math. Chem. 59 (2021) 1098-1116.

[7] Milano Chemometrics \& QSAR Research Group, Molecular Descriptors: the free online resource, Milano Chemometrics and QSAR Research Group, http://www. moleculardescriptors.eu/dataset/dataset.htm.

[8] K. C. Das, A. S. Cevik, I. N. Cangul, Y. Shang, On Sombor index, Symmetry 13 (2021) \#140.

[9] H. Deng, Z. Tang, R. Wu, Molecular trees with extremal values of Sombor indices, Int. J. Quantum. Chem., in press. 
[10] I. Gutman, Geometric approach to degree-based topological indices: Sombor indices, MATCH Commun. Math. Comput. Chem. 86 (2021) 11-16.

[11] I. Gutman, Some basic properties of Sombor indices, Open J. Discr. Appl. Math. 4 (2021) 1-3.

[12] A. Ghalavand, A. R. Ashrafi, Extremal graphs with respect to variable sum exdeg index via majorization, Appl. Math. Comput. 303 (2017) 19-23.

[13] A. Ghalavand, A. R. Ashrafi, Ordering of c-cyclic graphs with respect to total irregularity, J. Appl. Math. Comput. 63 (2020) 707-715.

[14] A. Ghalavand, A. R. Ashrafi, On forgotten coindex of chemical graphs, MATCH Commun. Math. Comput. Chem. 83 (2020) 221-232.

[15] A. Ghalavand, A. R. Ashrafi, Ordering chemical graphs by Randić and sumconnectivity numbers, Appl. Math. Comput. 331 (2018) 160-168.

[16] A. Ghalavand, A. R. Ashrafi, R. Sharafdini, O. Ori, Extremal chemical trees with respect to hyper-Zagreb index, Pure Appl. Math. 26 (2019) 177-188.

[17] I. Gutman, A. Ghalavand, T. Dehghan-Zadeh, A. R. Ashrafi, Graphs with smallest forgotten index, Iran. J. Math. Chem. 8 (2017) 259-273.

[18] B. Horoldagva, C. Xu, On Sombor index of graphs, MATCH Commun. Math. Comput. Chem. 86 (2021) 703-713.

[19] Y. Jiang, X. Chen, W. Lin, A note on chemical trees with maximal inverse sum indeg index, MATCH Commun. Math. Comput. Chem. 86 (2021) 29-38.

[20] Z. Lin, On the spectral radius and energy of the Sombor matrix of graphs, arXiv:2102.03960v1.

[21] X. Li, Z. Wang, Trees with extremal spectral radius of weighted adjacency matrices among trees weighted by degree-based indices, Linear Algebra Appl. 620 (2021) 6175.

[22] H. Liu, L. You, Z. Tang, J. B. Liu, On the reduced Sombor index and its applications, MATCH Commun. Math. Comput. Chem. 86 (2021) 729-753.

[23] I. Milovanović, E. Milovanović, M. Matejić, On some mathematical properties of Sombor indices, Bull. Int. Math. Virtual Inst. 11 (2021) 341-353.

[24] I. Redžepović, Chemical applicability of Sombor indices, J. Serb. Chem. Soc., in press. 
[25] T. Réti, T. Došlić, A. Ali, On the Sombor index of graphs, Contrib. Math. 3 (2021) 11-18.

[26] Z. Wang, Y. Mao, Y. Li, B. Furtula, On relations between Sombor and other degreebased indices, J. Appl. Math. Comput., in press. 